\title{
ЦИТ: ua217-057
}

DOI: 10.21893/2415-7538.2017-06-1-057

УДК 621.396.9

Набилков В.Д., Фомичев Н.И., Беляев А.А.

РАЗРАБОТКА И СОЗДАНИЕ ОБРАЗЦА АВТОМАТИЗИРОВАННОГО ИЗМЕРИТЕЛЬНОГО СТЕНДА ИЗМЕРЕНИЯ ДИАГРАММ НАПРАВЛЕННОСТИ АНТЕННЫХ СИСТЕМ

Ярославский государственный университет им. П.Г. Демидова, Ярославль, Советская 14, 150003

Nabilkov V.D., Fomichev N.I., Belyaev A.A. DESIGN AND DEVELOPMENT OF THE AUTOMATED MEASURING COMPLEX FOR ANTENNA MEASUREMENTS

P.G. Demidov Yaroslavl State University, Yaroslavl, Sovetskaya 14, 150003

Аннотация. В работе рассматривается структурная схема аппаратнопрограммного комплекса для проведения антенных измерений в ближней $u$ дальней зонах в диапазоне частот 1-12 ГГи. Дано описание алгоритма функииионирования разработанного программного обеспечения. Программное обеспечение представлено в виде графического пользовательского интерфейса u позволяет управлять измерительным оборудованием, устройствами позиционирования и выполнить пересчет диаграмм направленности из ближней зоны в дальнюю. Представлены результаты измерения амплитуднофазового распределения тестовой антенны в ближней зоне и диаграммы направленности на частоте 12 ГГи.

Ключевые слова: автоматизачия, измерения, диаграмма направленности

Abstract. In the paper a structural scheme of the complex for performing antenna measurements in the near and far zones in the frequency range 1-12 GHz is considered. A description of the algorithm of functioning of the developed software is given. The software is presented in the form of a graphical user interface and allows someone to control measuring equipment, positioning devices and perform recalculation of radiation patterns from the near zone to the far zone. The results of measurements of the amplitude-phase distribution of the test antenna in the near zone and the directivity pattern at a frequency of $12 \mathrm{GHz}$ are presented.

Key words: automation, measurements, antenna pattern

Вступление.

Целью работы является разработка аппаратно-программного комплекса для проведения автоматизированных измерений антенных систем в диапазоне $1-12$ ГГц.

В ходе выполнения работы были поставлены задачи: разработка структурной схемы аппаратно-программного комплекса, разработка алгоритмов управления и программного обеспечения для функционирования блоков комплекса.

Основной текст.

Аппаратно-программный комплекс (АПК) (рис.1) состоит из 
автоматизированного рабочего места (АРМ), планарного сканера, опорноповоротного устройства (ОПУ). Структурная схема строится по известным принципам, изложенным в [1]. АРМ предназначено для управления комплексом, отображения и протоколирования полученных результатов измерения. Планарный сканер позволяет производить измерение поля в ближней зоне с дальнейшим пересчетом результатов измерения в дальнюю зону. Опорно-поворотное устройство предназначено для проведения измерений в дальней зоне. АРМ представлено в виде персонального компьютера с установленным специальным программным обеспечением, подключенного к коммутатору ЛВС. Через коммутатор ЛВС происходит взаимодействие с основными блоками АПК - контроллером планарного сканера, контроллером опорно-поворотного устройства, векторным анализатором цепей.

Отличительной особенностью является использование векторного анализатора цепей для получения данных, соединенного с блоками контроллеров планарного сканера и ОПУ линией синхронизации.

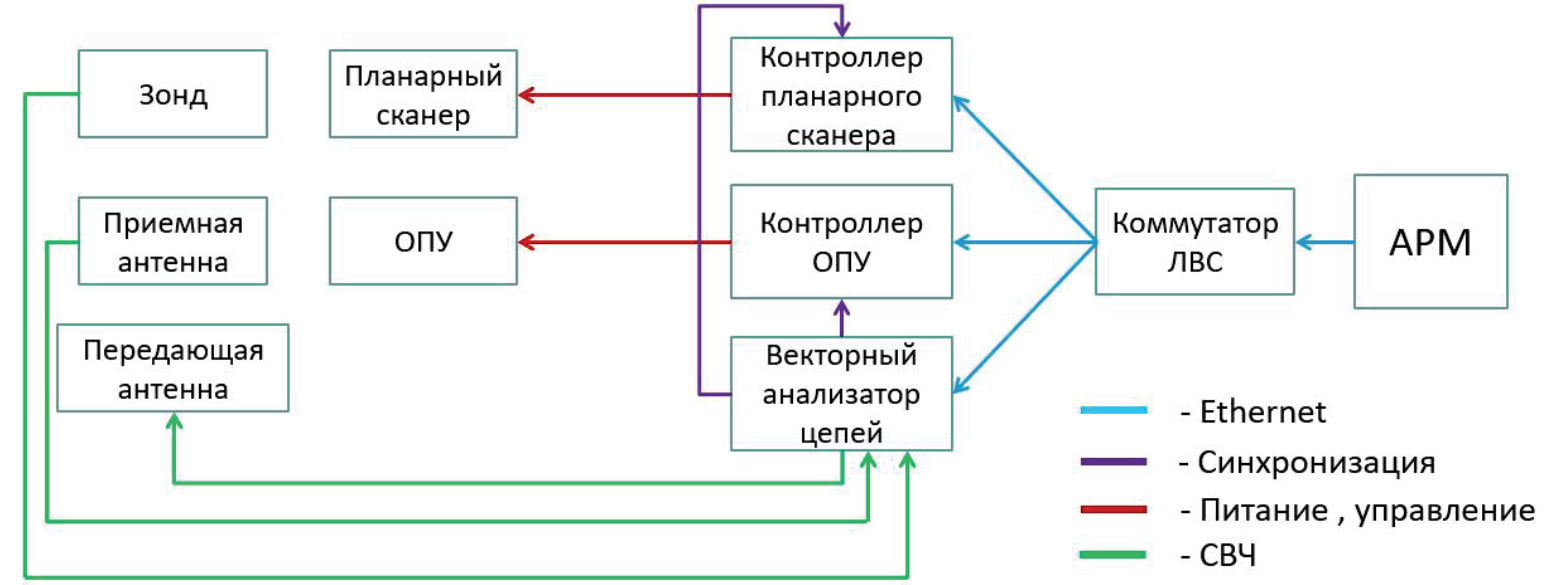

Рис. 1. Структурная схема АПК

Контроллеры передают анализатору цепей сигналы синхронизации о том, что необходимо производить измерения. Также в качестве сигналов синхронизации на АРМ через локальную сеть передаются пакеты данных, сообщающие о том, что данные с анализатора цепей необходимо принимать. В зависимости от выбора метода измерений к анализатору цепей подключаются передающая антенна, зонд, или приемная антенна. Взаимодействие блоков АПК основано на программном обеспечении.

Программное обеспечение представлено в виде графического пользовательского интерфейса, реализующего в себе такие функции как управление радиоизмерительным оборудованием, управление планарным сканером/опорно-поворотным устройством, регистрация и вывод результатов измерений. Программа управления АПК функционирует в соответствии с алгоритмом, представленным на рис. 2.

После запуска программы производится поиск необходимого для работы АПК оборудования в сформированной локальной сети с последующей идентификацией. После завершения идентификации программа ожидает формирование задания настроек для оборудования, таких как диапазон частот, 
шаг сканирования, скорость перемещения и др. Когда пользователь сформирует настройки оборудования, программа исходя из полученных данных о настройках, связанных с позиционированием, сформирует координатную сетку, согласно которой будет перемещаться сканер или платформа ОПУ. Далее следует цикл измерений. Одна итерация цикла измерений выглядит следующим образом (рис. 3).

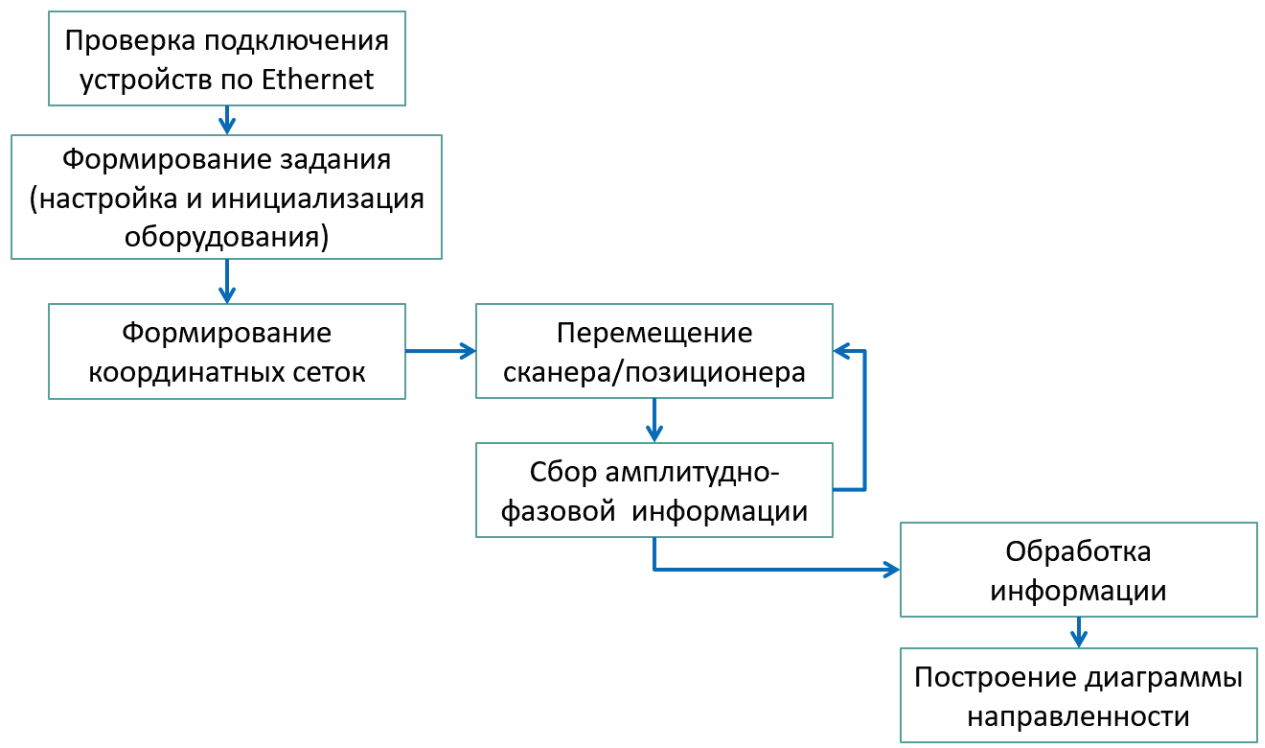

Рис. 2. Алгоритм управления АПК

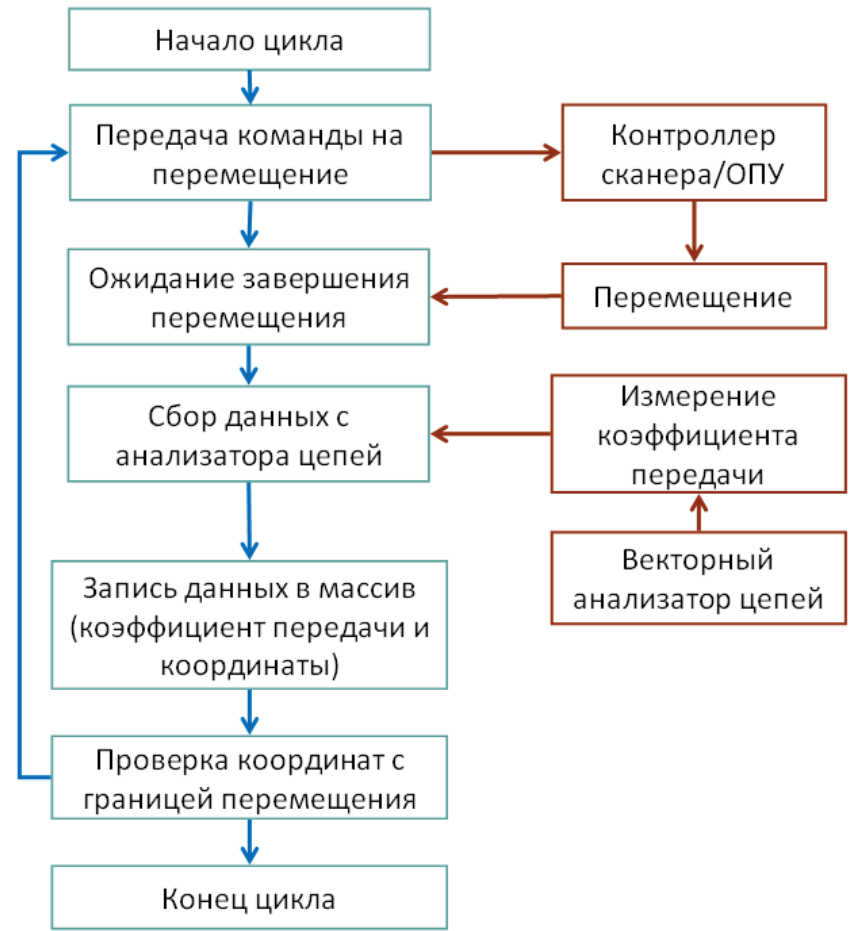

\section{Рис. 3. Цикл измерений}

По завершению цикла измерений полученный массив данных со значением комплексного коэффициента передачи и соответствующей коэффициенту координатой подвергается обработке (в случае с методом измерения в ближней зоне пересчитывается в дальнюю). Разработанное 
программное обеспечение реализовано на объектно-ориентированном языке программирования высокого уровня $\mathrm{C}++$, в среде разработки Qt. Для управления оборудованием применяется стандарт взаимодействия с измерительными приборами SCPI.

Для проверки работоспособности комплекса проведены тестовые антенные измерения. В качестве измеряемой антенны выступала широкополосная измерительная рупорная антенна П6-123 на базе Н-образного волновода.

На рис. 4 и 5 представлены амплитудное и фазовое распределение поля в ближней зоне на частоте 12 ГГц.

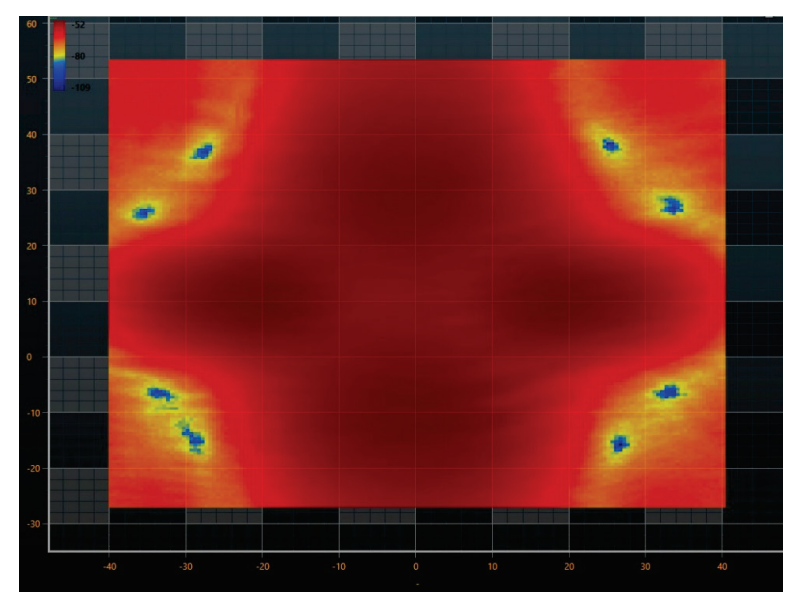

Рис. 4. Амплитудное распределение

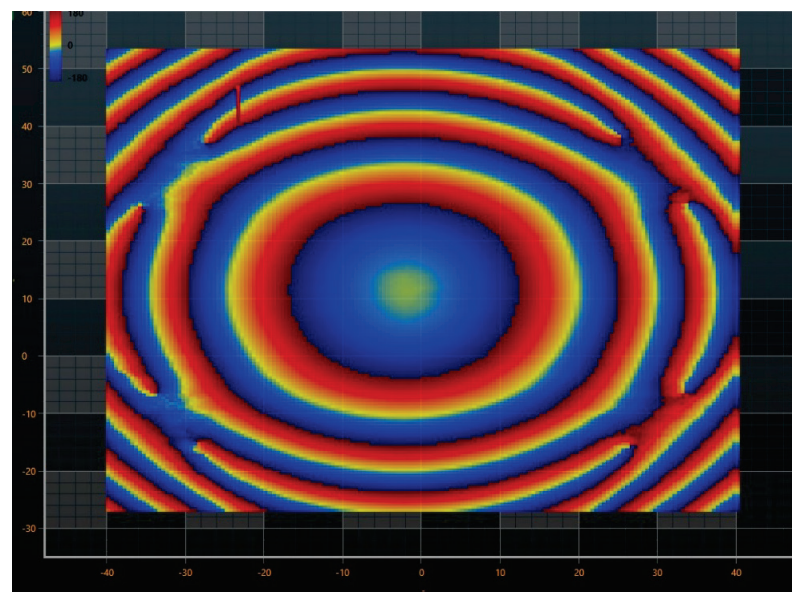

Рис. 5. Фазовое распределение

По полученным данным о распределении поля в ближней зоне был произведен пересчет диаграммы направленности в дальнюю зону. Результат пересчета представлен на рис. 6.

Измерения были проведены в безэховой камере размером 12x4.2 м, что позволяет обеспечить точности проведения измерений.

\section{Заключение и выводы.}

Таким образом, разработан и реализован аппаратно-программный комплекс, позволяющий проводить измерения диаграмм направленности антенных систем в дальней и ближней зоне, в диапазоне от 1 до 12 ГГц. Комплекс располагается на базе безэховой камеры, что обеспечивает точность 
проводимых измерений. Разработанное программное обеспечение позволяет выполнить пересчет диаграмм направленности из ближней зоны в дальнюю.

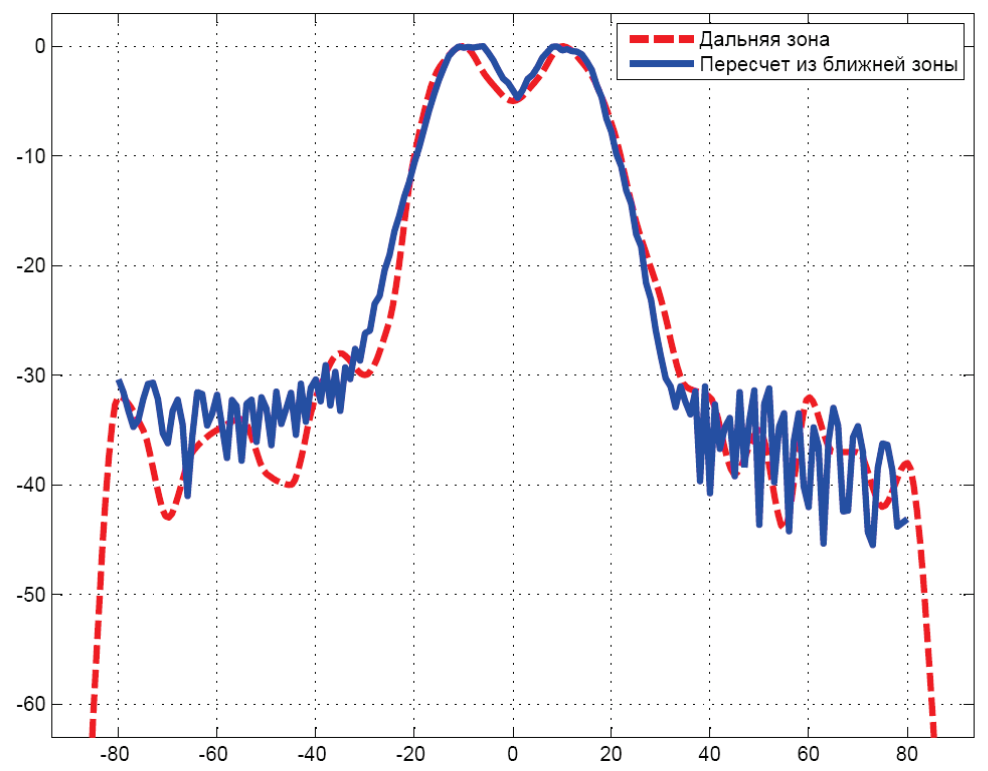

Рис. 6. Диаграмма направленности в дальней зоне

Литература:

1. Захарьев Л.Н, Леманский А.А., Турчин В.И., Цейтлин Н.М., Щеглов К.С. Методы измерения характеристик антенн СВЧ / Издательство «Радио и связь», 1985. С. $71-128,136-206$

2. Бахрах Л.Д. и др. Методы измерений параметров излучающих систем в ближней зоне. Л.: Наука, 1985.

Статья отправлена: 06.06.2017

(C) Набилков В.Д., Фомичев Н.И., Беляев А.А.

ЦИТ: иа217-046

DOI: 10.21893/2415-7538.2017-06-1-046

УДК 608.2

Окулов В.И., Пуляев И.А. ПЕРСПЕКТИВЫ ПРИМЕНЕНИЯ ДВИГАТЕЛЯ СТИРЛИНГА НА СОВРЕМЕННОМ ЭТАПЕ

Одесский национальный морской университет,

Одесса, Мечникова 34, 65029

Okulov V.I., Pulyaev I.A.

PROSPECTS OF APPLICATION OF STIRLING ENGINE AT THE PRESENT STAGE

Odessa National Maritime University,

Odessa, Mechnikova 34, 65029

Аннотация. В статье проводится анализ применения в настоящее время двигателей Стирлинга, и даются рекомендащии по наиболее перспективным 